\title{
Development of a Knowledge Management System for Energy Driven by Public Feedback
}

\author{
Massimiliano Fratoni, Joonhong Ahn, Brandie Nonnecke, \\ Giorgio Locatelli and Ken Goldberg
}

\begin{abstract}
The Nuclear Engineering Department at the University of California, Berkeley, in collaboration with the Industrial Engineering and Operations Research Department and the University of Lincoln in the United Kingdom, is proposing to create an open web platform that makes high-quality scientific data on energy sources readily available, assembles those data into metrics more suitable to the general public's knowledge and interest (e.g. impact on the family's budget or green house gas emission), and visually renders such information in a straightforward manner.
\end{abstract}

Keywords Knowlegement management - Web platform • Metrics • Nuclear energy

\section{Introduction}

In the era of information technology a large amount of data is readily available at everyone's fingertips. Energy and its implications, scarcity or abundance of resources, impact on climate change, emissions of pollutants, and more are topics of global interest that receive strong attention across all media. Opinions, official statements, and scientific data create a continuous flow of information. Nuclear energy among all sources is the subject of strong debates with cohorts of supporters and detractors ready to pinpoint its benefits or its drawbacks, respectively. In this large pool of information, it is of paramount difficulty even for field experts to isolate scientific data on energy, and to select reliable and coherent sources. Furthermore, higher quality data are often packaged in scientific jargon and are presented in forms and ways to which the general public does not relate

M. Fratoni $(\bowtie) \cdot$ J. Ahn $\cdot$ B. Nonnecke $\cdot$ K. Goldberg

University of California, Berkeley, CA, USA

e-mail: maxfratoni@berkeley.edu

G. Locatelli

University of Lincoln, Lincoln, UK

(C) The Author(s) 2017

J. Ahn et al. (eds.), Resilience: A New Paradigm of Nuclear Safety,

DOI 10.1007/978-3-319-58768-4_10 
(e.g. investment NPV, Sox produced, GDP impact, etc.). The Nuclear Engineering Department at the University of California, Berkeley, in collaboration with the Industrial Engineering and Operations Research Department and the University of Lincoln in the United Kingdom, is proposing to create an open web platform that (1) makes high-quality scientific data on energy sources readily available, (2) assembles those data into metrics more suitable to the general public's knowledge and interest (e.g. impact on the family's budget or green house gas emission), and (3) visually renders such information in a straightforward manner. Through this platform users will be able to create "energy portfolios" by mixing energy sources and evaluating how different choices impact the metrics they are interested in. Rather than a top-down approach, the platform will solicit feedback from the end-user on the prioritized topics as well as contribute additional topics with help of a knowledge management system.

\section{Functionalities of the Envisioned Platform}

The proposed web platform will include two major components: a user opinion component with working name "Energy Report Card" and an information component with working name "The Energy Challenge".

The "Energy Report Card" integrates elements from the Opinion Space project (http://opinion.berkeley.edu/) and the California Report Card project (http:// californiareportcard.org) developed at the CITRIS Data and Democracy Initiative and informed by work done by the World Bank on the use of report cards as assessment tools of government performance. The Energy Report Card gathers feedback on users' perceptions toward environmental, social, and economic impacts of energy sources. Upon entering the system users will be asked to assign a value from 0 "Strongly Disagree" to 9 "Strongly Agree" on six quantitative assessment questions that will be used to gauge each user's preference for environmental, social, and/or economic impacts as high priority issues (Fig. 1). For example, participants will be asked whether they believe global warming (environmental impact) is a high priority issue, whether job creation (social impact) from energy production is a high priority issue, and whether energy cost stability (economic impact) is a high priority issue, among others.

Participants will then enter "The Energy Challenge" where they will be presented with an energy portfolio that matches their personal environmental, social, and economic interests. Participants will be able to adjust the different energy sources composing their energy portfolio. As they add and remove components to the portfolio they can observe how the selected metrics respond to each change. Additional text, graphics, videos, and links will also be provided through the page to explain the correlations between sources and metrics (similarly to what is done in the "California Budget Challenge"). Unrealistic scenarios, i.e. 100\% nuclear energy or $100 \%$ solar energy, will prompt a warning message with an accurate and straightforward explanation of why such scenarios are unrealistic. A visual 


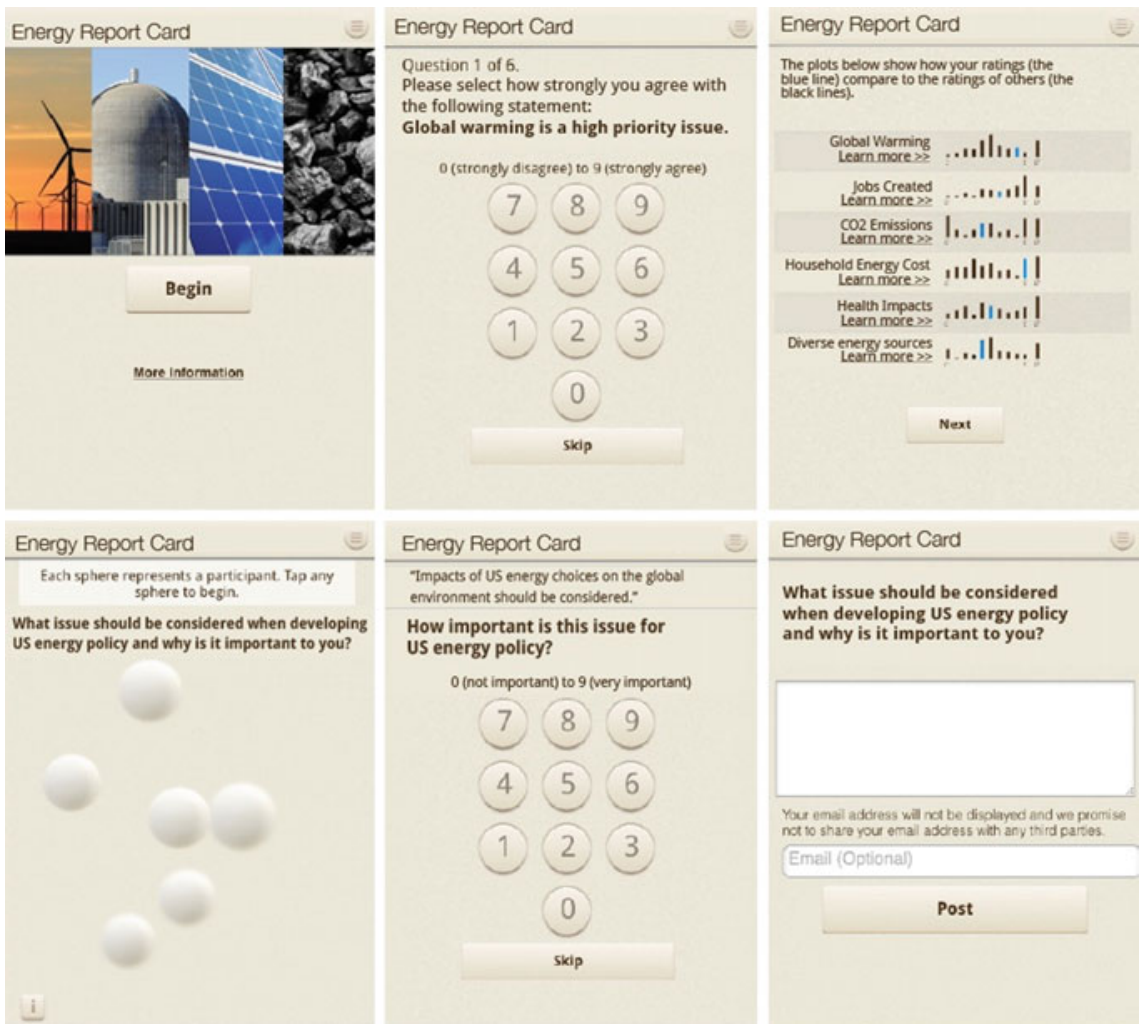

Fig. 1 Example of the structure and functionalities of the "Energy Report Card". The panels from left to right, top to bottom show: introductory panel; example of quantitative assessment; individual versus average assessment distribution; 2-D Principal Component Analysis display; assessment of opinions of other users; user input panel. This example was adapted from the "California Report Card" and actual name, content, metrics, functions, and graphics will be developed as part of the proposed project

rendering system will be developed to visualize the outcome of the users' choices in intuitive ways. For example, users could choose to visualize a comparison of the volume of waste created by each source, or visualize the fraction of US territory that needs to be used for each source on a US map. Users will finally have the option to share their personalized energy portfolio and metrics of choice through email and social media.

After completing "The Energy Challenge", participants will then enter the final portion of the "Energy Report Card" where they will be able to suggest additional issues they believe are important to consider when designing an energy portfolio. Participants will also rate the importance of others' suggestions, enabling crowd-sourced insights. We apply Principal Component Analysis (PCA) to display each participant's suggestion on a two-dimensional plane. Each user is represented 
in the system by a sphere (see bottom left panel in Fig. 1). To avoid overcrowding, we load only a few spheres onto the plane at a time. In a first step, we associate each user with a k-dimensional vector: one entry corresponding to each response to the assessment questions. We then apply PCA to the set of vectors and the algorithm returns a two dimensional $(\mathrm{x}, \mathrm{y})$ location for each participant. This point corresponds to the top 2 eigenvectors of the covariance matrix. We then center the visualization on the user's (xp, yp) position, and then arrange the spheres in the new coordinate space. Spheres in closer proximity represent users who responded to the assessment questions similarly. This allows users to immediately see how people similar to them feel about what issues should be considered when developing an energy portfolio. Spheres that are larger in size represent users whose suggestion has been rated as highly important by others.

\section{Evaluation Metrics}

The metrics that we will use to gauge public perception of energy and its sources must be familiar to the general public rather than technical. At the same time the significance and relevance of such metrics will be guaranteed following a well-established framework. The United Nations World Commission on Environment and Development (WCED) in the 1987 defined sustainable development as the "development that meets the needs of the present without compromising the ability of future generations to meet their own needs." [1] A typical framework, empowering this definition is the "Triple Bottom Line" [2]. The Triple Bottom Line (3BL) is a framework, well established in the scientific literature as well as public-oriented publications, with three key elements: social, environmental (or ecological) and economics. It provides a holistic perspective to assess the sustainability of several engineering solutions. A state-of-the-art framework to assess the sustainability of power plants and their life cycle (nuclear in particular) is provided in [3]. Regarding environmental indicators in particular, the US EPA has focused on determining and developing the best impact assessment tool for Life Cycle Impact Assessment (LCIA), Pollution Prevention (P2), and Sustainability Metrics for the US. This research led to the creation of TRACI-the Tool for the Reduction and Assessment of Chemical and other environmental Impacts. The methodology has been developed specifically for the US using input parameters consistent with US locations. Site specificity is available for many of the impact categories, but in all cases a US average value exists when the location is undetermined. The average values were implemented in the ecoinvent data. Further information is available at http://www.epa.gov/nrmrl/std/traci/traci.html. TRACI is therefore useful to compare different power plants and their life cycle. Unfortunately, these frameworks are hardly compressive for non-experts. In particular regarding the power sectors, people often have misconceptions that the tool envisaged by this research program will contribute to overcome. Some of the most relevant examples that we will address are: 
(1) Thinking at technology level is inappropriate

- The same technology has different performances in different scenarios: e.g. technology X can have great performance in scenario A (desert with plenty of sun), poor performance in scenario B (north country with several rainy days).

- An electrical system to work in an efficient way (from technical and economical perspectives) needs the right mix of power plants: base load, peak load, ancillary services, etc.

(2) Energy cost is just one aspect of economics

- People need to distinguish between Production Cost (technology driven), Electricity Price (market driven) and Value (usage driven). Gas turbines working as spinning reserve are costly, get a high price, but are extremely valuable. A private company working in a market has, usually, the goal to maximize profits minimizing risk, not minimize production costs.

- Let us assume that technology A has an overall production cost (LUEC) of $\$ 100$ per MWe and B $\$ 70$ per MWe. Is B better than A? We need to include environmental issues, but also social. Let's think about social. Maybe $\mathrm{B}$ is not creating local national jobs, while $\mathrm{B}$ is more expensive, but the cost is boosting local/national economics.

(3) Global warming

- The majority of scientific publications say it is an issue. However, we still lack understanding of how citizens feel about global warming and their preferences for dealing with it. In a world (or nation) with limited resources it is important to prioritize budget allocations for important social, economic, and environmental issues. Identifying how citizens would allocate limited resources could provide insights into citizens' feelings toward global warming. For example, having $\$ 100$ to invest-how much should be allocated to "cutting greenhouse gas emission", "funding cancer research", "paying for vaccinations in poor countries", "creating grants for student education", and "developing more sustainable food production techniques"?

This research leverages the state-of-the-art knowledge to create an innovative social engagement platform that will allow for key insights to emerge on public perception toward different energy sources, including perceptions toward different environmental, social, and economic impacts. The 3BL elements can be broken down into categories (and eventual sub-categories) and the categories in quantitative indicators. This framework, common for all the energy sources, differs for the specific values of each indicator, specific for the source considered. The key idea is to use indicators that are intuitive for the "average citizen". This indicator requires a "life cycle perspectives" and needs to be tuned from existing research and database (e.g. http://www.externe.info/). In this way the user can focus his/her attention on specific aspects. 
We will give the option to the user to assign "weight" to different categories to obtain the "ideal ranking". For example, an "Environmentally sensitive user" can assign a high importance to the environmental indicators and/or categories and the system will return an energy portfolio that reflects these interests. There is precise set of mathematical methods to address in an exact way this issue, and they are built around the Multi Attribute Decision Making theories. The Analytic hierarchy process (AHP) is rather simple and straightforward [3], but if there are interactions between categories it is better to use the Analytic Network Process (ANP) [4]. The system, receiving the input from the user, will apply these methods for the ranking of different energy-mix alternatives.

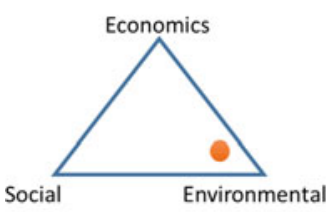

Extreme environmental activist

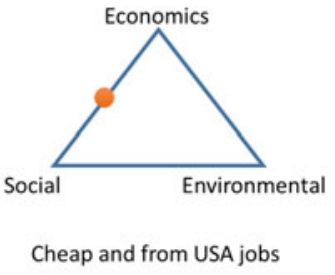

Cheap and from USA jobs

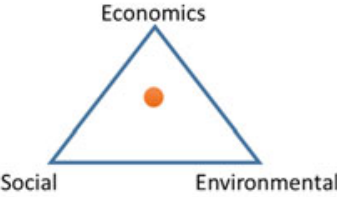

The pragmatic view

shows an example of three possible choices from three different users. This system will record the choice of each user and will display. The overall ranking calculated from all users. This information, "the voice of the average citizen", will be of paramount importance paving the way for research and policy decision-making in the energy sector. At the present time there is very limited understanding about how the public addresses trade-offs between the different 3BL elements and which indicators are more relevant. Moreover, users will be asked to provide demographic information (e.g. zip code, gender, age, education), allowing for more in-depth analyses. This "feedback data" will be released in a public user-friendly way for the benefit of the public, policymakers and the scientific community.

\section{Discussion}

We expect that the development of a web platform for comparing energy sources through easy-to-relate-to metrics will promote dialogues between experts and the general public, and will enable exploration and visualization of the public's points of interests, so that the policymakers can correctly understand the needs and priorities of their constituents. Unlike typical top-down approaches with predefined recipes and query items, the proposed system lets the end-user prioritize metrics of interest, provide additional metrics not originally included, provide suggestions and evaluate other users' ideas. While such sense of trust is sought providing technically 
reliable data sources and models, interpretation into a straightforward rather than technical language is essential.

This platform will implement best practices derived from similar existing efforts like "my2050", but it will largely depart from the underlying philosophy of such tools. We strongly believe that a visually attractive platform is necessary to attract users to engage with critical energy issues. Nevertheless, the success of the platform will be determined by the rate at which users return to the platform and make constant use of it. The unique features that we propose allow users to express their opinions and concerns, and to understand the impact of their choices on easy-torelate-to metrics. We expect that the personalization aspect and the focus on the user's interest, rather than providing a pre-packaged solution, will make the user want to come back and bring other users to the platform. Furthermore, energy policymakers in general will want also to come back to the site and continuously monitor it as data and metadata evolves with time and events. A transparent interface with social media will further facilitate users' participation.

\section{References}

1. World Commission on Environment and Development, Our Common Future (Oxford University Press, Oxford Paperbacks, 1987)

2. J. Elkington. Cannibals with Forks: The Triple Bottom Line of 21st Century Business (New Society Publishers, 1998)

3. T.L. Saaty, What is the Analytic Hierarchy Process? (Springer, Berlin Heidelberg, 1988)

4. T.L. Saaty, Decision Making with Dependence and Feedback: The Analytic Network Process (RWS Publications, 1996)

Open Access This chapter is licensed under the terms of the Creative Commons Attribution 4.0 International License (http://creativecommons.org/licenses/by/4.0/), which permits use, sharing, adaptation, distribution and reproduction in any medium or format, as long as you give appropriate credit to the original author(s) and the source, provide a link to the Creative Commons license and indicate if changes were made.

The images or other third party material in this chapter are included in the chapter's Creative Commons license, unless indicated otherwise in a credit line to the material. If material is not included in the chapter's Creative Commons license and your intended use is not permitted by statutory regulation or exceeds the permitted use, you will need to obtain permission directly from the copyright holder.

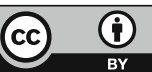

